\title{
Experiencia de Formación y Promoción de Emprendedores Tecnológicos desde una Carrera Universitaria
}

\author{
Roberto Muñoz \\ Departamento Ingeniería en Sistemas de Información \\ Universidad Tecnológica Nacional, Facultad Regional Córdoba, \\ Córdoba \\ director@frc,utn.edu.ar

\section{Oscar Carlos Medina} \\ Departamento Ingeniería en Sistemas de Información \\ Universidad Tecnológica Nacional, Facultad Regional Córdoba, \\ Córdoba \\ omedina@frc.utn.edu.ar
}

\section{Carolina Molina}

Departamento Ingeniería en Sistemas de Información

Universidad Tecnológica Nacional, Facultad Regional Córdoba,

Córdoba

molinacarolinaa@gmail.com

\section{Gabriela Alexis Falcón}

Departamento Ingeniería en Sistemas de Información

Universidad Tecnológica Nacional, Facultad Regional Córdoba, Córdoba

inggabrielafalcon@gmail.com

\section{Resumen}

Este trabajo describe al programa de Mentoreo de Emprendedores de la Carrera Ingeniería en Sistemas de Información que se desarrolla en la Universidad Tecnológica Nacional - Facultad Regional Córdoba. Con el propósito de complementar el perfil profesional de los ingenieros de esta Facultad se creó un nuevo espacio académico convocando a estudiantes y graduados con espíritu emprendedor y proyectos innovadores que deseen crear empresas de base tecnológica. El ingreso al programa es voluntario e incluye actualmente tanto a emprendedores 
como a mentores graduados y docentes. Dentro del mismo se capacita y fomenta la innovación, la cultura emprendedora y la inserción en el ecosistema emprendedor local. De acuerdo a los resultados alcanzados se prevé una proyección positiva a mediano plazo y la consolidación de un modelo de trabajo que permita su escalabilidad y replicación.

Palabras Clave: Emprendedor tecnológico, Carrera universitaria, Mentor, Emprendimiento.

\begin{abstract}
This paper describes the Entrepreneurs Mentoring program of the Engineering in Information Systems Career that is dictated in the Universidad Tecnológica Nacional - Facultad Regional Córdoba. With the purpose of complementing the professional profile of the engineers of this Faculty a new academic space was created summoning to students and graduates with enterprising spirit and innovative projects that wish to create companies of technological base. Admission to the program is voluntary and currently includes entrepreneurs as well as graduate mentors and professors. Within it, training and innovation, entrepreneurship culture and insertion in the local entrepreneurial ecosystem are trained and promoted. According to the results achieved, a positive projection is foreseen in the medium term and the consolidation of a work model that allows its scalability and replication.
\end{abstract}

Keywords: Technological entrepreneur, University career, Mentor, Entrepreneurship.

\title{
Introducción
}

Las experiencias en formación de emprendedores latinoamericanos de los últimos años se da en estructuras académicas y administrativas de variada naturaleza, "los centros y programas se constituyen en los de mayor presencia y relevancia, y la docencia su principal actividad" (Paz et al., 2014) cuya estrategia pedagógica más relevante se basa en el aprender haciendo privilegiando la formación del emprendedor empresario. Las estrategias más utilizadas se centran en los métodos de aprendizaje experiencial ya que "el modelo pedagógico que se requiere para la formación del profesional emprendedor no se puede encuadrar en una teoría única, más bien es el resultado de la interacción de varias de ellas. Se caracterizan por el papel activo que toma el estudiante en el proceso de aprendizaje, valoran el papel del error como potenciador del conocimiento y propenden por hacer de la educación una experiencia transformadora" (Fracica, 2009). La conjunción en esta tarea de factores tales como son la formación, la innovación y el desarrollo tecnológico le otorgan un papel clave a las Universidades Tecnológicas para desempeñar. Así lo expresa el Tecnológico de Monterrey: "La convicción de que la universidad es uno de los principales promotores del desarrollo socioeconómico regional por su capacidad para incrementar la competitividad internacional basada en el conocimiento, 
la innovación y el desarrollo tecnológico, con responsabilidad social y ambiental" (González González \& López Preciado, 2012). Sin embargo "el emprendimiento no se ha incorporado plenamente en la filosofía institucional" (Paz et al., 2014) de las carreras de grado en nuestro país. Cabe destacar dentro de un marco de referencia latinoamericano, se espera que la Universidad sea uno de los actores involucrados en el fomento de la actividad emprendedora junto con "las agencias de apoyo a la MiPyME, los ministerios de trabajo, las agencias de formación profesional, los centros de formación superior y las cámaras, gremios y organizaciones no gubernamentales dedicadas al desarrollo del sector privado" (Angelelli \& Prats, 2005).

Con estas premisas en la planificación estratégica del Departamento Ingeniería en Sistemas de Información de la Facultad Regional Córdoba de la Universidad Tecnológica Nacional se incluye un programa trianual para el mentoreo de emprendimientos de base tecnológica partiendo de proyectos innovadores de estudiantes y graduados de la Carrera que voluntariamente acepten el desafío de emprender, cuyos primeros resultados se detallarán a continuación.

\section{Programa de mentoreo de emprendedores ISI}

La carrera de Ingeniería en Sistemas de Información, de la Universidad Tecnológica Nacional, proporciona, en su diseño curricular, asignaturas que permiten formación y prácticas para la generación de proyectos innovadores como ser "Proyecto Final" y en el caso particular de la Facultad Regional Córdoba ha implementado, en el tramo de asignaturas electivas, las propuestas de "Creatividad e innovación en Ingeniería” y "Emprendimientos tecnológicos”. Por su parte la asignatura "Habilitación Profesional", de tercer año, y obligatoria sólo para el título intermedio de Analista Universitario de Sistemas, exige la presentación de un proyecto de desarrollo. Pero además, por la impronta que le supieron dar los docentes y su contenido, se debe agregar en esta lista a la cátedra de "Comunicaciones", de tercer año, donde se generan trabajos prácticos multidisciplinarios y de innovación tecnológica.

Este conjunto de proyectos se hizo visible en congresos académicos, en eventos de tecnología de información y en los medios de comunicación locales. A partir de estas iniciativas se detectó la necesidad de crear un espacio que pudiera extenderse más allá de una asignatura brindada en las aulas, que provea los conocimientos teóricos y el acompañamiento en las actividades de negocio iniciales, de manera que puedan minimizar los errores y tener más posibilidades de éxito al transformar un trabajo académico innovador en un emprendimiento de base tecnológica.

El logo del programa que muestra la Figura 1 pone de manifiesto el trípode en el que se basan las actividades de mentoreo: la Universidad Tecnológica Nacional, representada por su isologo, la Innovación y la Tecnología representadas por la lámpara de luz encendida. 


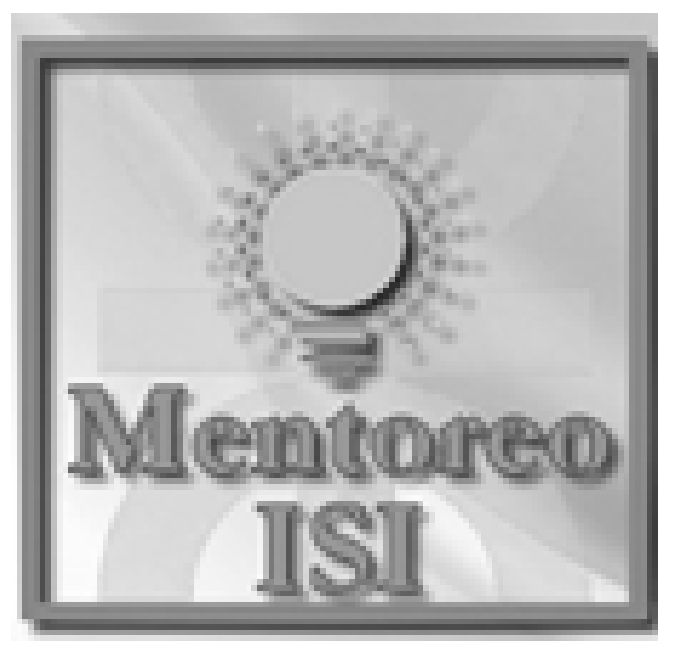

Figura 1: Logo de Mentoreo ISI

Con este logo como emblema y guía fue planteado el programa de "Mentoreo de Emprendedores ISI” en el Plan Estratégico de la Carrera Ingeniería en Sistemas de Información, de la Universidad Tecnológica Nacional, Facultad Regional Córdoba, para el período 2015-2017, en la búsqueda de apoyar a los estudiantes y recientes graduados de la Carrera que decidan insertar en el mercado su primer emprendimiento basado en un trabajo académico.

En primera instancia se llevó a cabo un plan de capacitación del personal docente que se involucraría en las actividades y se fortalecieron vínculos con sectores provinciales y nacionales, públicos y privados, del ecosistema emprendedor. El ecosistema emprendedor local por otra parte goza de un interés público creciente, citando al Lic. Lawson: "Córdoba es el país de los sueños, tiene uno de los mejores ecosistemas emprendedores del mundo, con todo tipo de organizaciones que acompañan todos los días a los miles de emprendedores que desean asumir la enorme responsabilidad de formar su propia empresa." (Bentolila et al., 2013), y a su vez aclara quiénes son a su entender los emprendedores: "Quienes sueñan con transformar una idea en una empresa, quienes creen en la importancia de las empresas para el desarrollo de la sociedad, quienes quieren asumir un rol de transformador en su pueblo o ciudad" (Bentolila et al., 2013).

Por ello el programa trabaja en la primera etapa de la fase temprana del proceso emprendedor, que comprende el momento en que el estudiante visualiza la oportunidad para desarrollar un emprendimiento comercial a partir de un trabajo innovador que junto con su grupo concretó en las aulas. A partir de esta decisión de emprender, se generan actividades, en forma individual y grupal, claves para que los mentoreados puedan concretar sus "sueños". Nuestro país tiene hoy la posibilidad efectiva de aumentar la creación de nuevos emprendimientos. Si bien Argentina tiene una tasa de natalidad de emprendimientos formales e informales creciente, en un contexto Latinoamericano "Argentina y México tienen tasas de entrada sustancialmente menores de lo que cabría esperar dado su PBI per cápita" (Lederman et al., 2014). Por ejemplo comparando con Costa Rica "cuya tasa de entrada, de casi 16 nuevas empresas cada 1.000 personas en edad de trabajar, cuadruplica el nivel de referencia internacional” (Lederman et al., 2014). 
Una de las actividades principales que se lleva a cabo en este espacio, es el soporte para la toma de las primeras decisiones de negocios. En esa etapa el mentor proporciona su visión y perspectiva, se debate con los emprendedores y son ellos quienes finalmente seleccionan la alternativa que consideran más conveniente. Cada decisión tomada va marcando un rumbo hacia el cumplimiento o no de los objetivos del emprendimiento, teniendo en cuenta que la validación real del producto o servicio ofrecido la realiza el mercado. Como ejemplo de esto podemos citar la oportunidad de lanzamiento del emprendimiento dentro de un espacio de trabajo compartido, comúnmente denominado "co-working" con un convenio firmado con la U.T.N., que los benefició a nivel económico, de relaciones comerciales y de cumplimiento de requisitos de financiamiento como lo es el aporte no reembolsable "Emprende INNdustria" (Gobierno de Córdoba, 2017).

Otra actividad importante es la vinculación que facilita a los emprendedores una amplia red de contactos con graduados, docentes y organizaciones que tienen firmado convenio con la Facultad, empresas y entidades del ecosistema emprendedor local con las que puedan interactuar estableciendo las primeras relaciones de negocios. Tal fue el caso de mediados del año 2015, cuando una videoconferencia con la empresa Microsoft Argentina, organizada por un graduado que trabaja allí, posibilitó a posteriori que dos emprendimientos pudieran darse a conocer en las redes sociales y medios de comunicación tradicionales en una campaña internacional de un nuevo producto de la empresa (Microsoft, 2017a), (Microsoft, 2017b), con la temática de promocionar innovaciones de impacto social.

Desde el año 2015 se mantienen canales de comunicación permanentes, para difundir novedades de eventos organizados en la Facultad, en el área temática de incumbencia, presencial o de realización virtual, agregando el valor de filtro para seleccionar el contenido que es pertinente para los emprendimientos en curso y disparadores de diálogos. Esto genera un espacio de retroalimentación, formación y discusión entre los mentores y mentoreados. Para que sea más ágil la actualización de los contenidos, las novedades se publican en la página institucional de la Carrera (ISI, 2017a), se envían por correo electrónica a una lista exclusiva conformada por mentores y mentoreados, una "fan page" de Facebook (ISI, 2017b) y una cuenta de Twitter (ISI, 2017c).

Por último, mediante capacitaciones y talleres grupales se impulsa el desarrollo de habilidades interpersonales e inteligencia emocional, capacidades de liderazgo, cambio y marketing personal, se comparten herramientas para la conceptualización de ideas de negocios y conformación de equipos de trabajo.

\section{Objetivos}

Los objetivos del programa en relación a los estudiantes y graduados mentoreados, que están publicados en la página institucional de la Carrera (ISI, 2017d), son los siguientes:

- Promover el Emprendedorismo y la Innovación tecnológica.

- Mentorear, capacitar y vincular a los grupos de estudiantes con proyectos innovadores que deseen transformarlos en emprendimientos tecnológicos. 
- Viabilizar la participación institucional de los estudiantes mentoreados en eventos de TIC, Emprendedorismo y Congresos de Informática / Sistemas de Información.

- Posibilitar el trabajo interdisciplinario con estudiantes y/o profesionales de otras Carreras, si el emprendimiento lo requiere.

- Colaborar con la formalización de los proyectos y planes de negocio para presentar a futuros inversores, convocatorias y programas de financiamiento.

- Facilitar el contacto con graduados emprendedores tecnológicos, espacios de trabajo compartido y organizaciones de soporte a PYMES y nuevos emprendimientos.

- Promover la publicación de los proyectos como artículos científicos y póster en congresos, eventos académicos y revistas científicas.

\section{Metodología y herramientas de trabajo}

La estrategia pedagógica tiene por finalidad integrar teoría y práctica, orientada fundamentalmente al "saber hacer", en cuanto a la creación de un emprendimiento de base tecnológica, caracterizándose por el aprendizaje por descubrimiento y trabajo en equipo. Se busca facilitar el acceso a conceptos, metodologías y herramientas que les permita, a los mentoreados, fundamentar científica, ingenieril y administrativamente un producto o servicio de base tecnológica, como resultado del trabajo individual y grupal que involucra la participación de todos sus integrantes.

El programa de mentoreo expande esta área temática como un complemento formativo de la Carrera y desarrolla habilidades de negocio e innovación, de manera que los emprendedores puedan contar con un mejor andamiaje que les permita concretar sus proyectos y recorrer con éxito los primeros pasos para insertarse en el mercado.

Los emprendedores deben poder generar su primer Plan de Negocios, con todos los componentes cualitativos y cuantitativos de un emprendimiento tecnológico, determinando la conveniencia o no del negocio y una presentación del proyecto abreviada en forma oral, lo que comúnmente se conoce como "elevator pitch". También se busca que logren elaborar artículos científicos, afiches, postulaciones a concursos y a programas de financiamiento.

La metodología anual de trabajo, que se grafica en la Figura 2, tiene inicio en febrero con la evaluación de los proyectos más destacados en las cátedras de la Carrera, por parte de los docentes en conjunto con el equipo de mentoreo que presentan este artículo. 


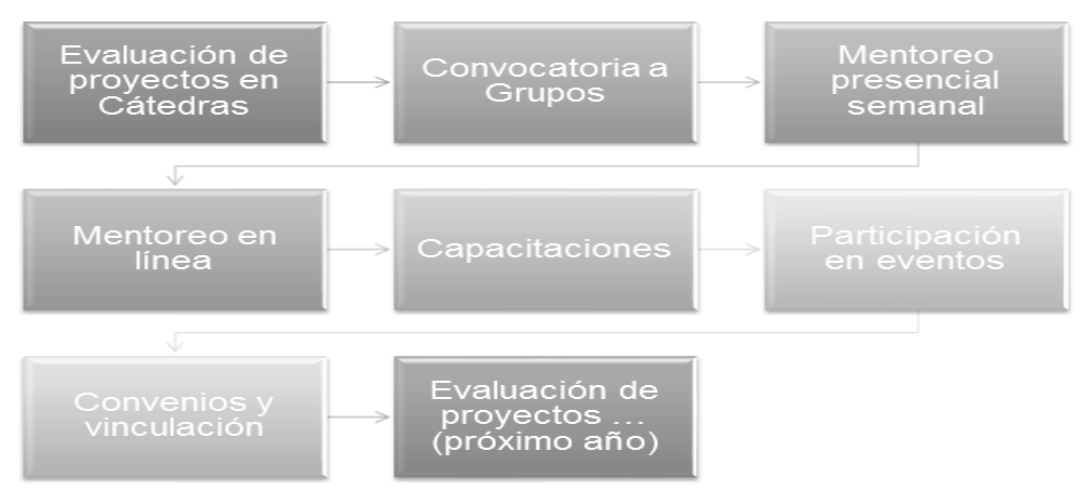

Figura 2: Metodología anual de Mentoreo ISI

Se contacta por correo electrónico a los integrantes de estos proyectos, más los emprendimientos mentoreados el año anterior, y se organiza un evento en la Facultad para formalizar la convocatoria y difundir el programa a toda la Comunidad. Con las respuestas positivas, de desear avanzar con el proyecto, se confecciona el padrón de mentoreados del año en curso y se actualiza la lista privada de correos electrónicos.

Sobre este padrón se organiza otro evento invitando a docentes y graduados a colaborar con los proyectos, cumpliendo el rol de mentores. Con la confirmación de los mentores se coordinan reuniones con los grupos hasta que cada mentor tenga asignado un emprendimiento. Cuando se haya logrado el consenso, se actualiza el padrón y la lista de correos agregando los mentores.

Conformados los equipos de trabajo con mentores y mentoreados, se realizan las siguientes actividades:

- Reuniones de Mentoreo en el Departamento Ingeniería en Sistemas de Información, una vez a la semana, y durante los otros días en modo "on line", a través de chat, correo electrónico y redes sociales.

- Dictado de conferencias de divulgación y capacitación en emprendedorismo y tecnologías de información, con publicación e inscripción "en línea" en la página institucional de la Carrera y entrega de certificados a los asistentes de las conferencias.

- Promoción de mentores docentes o graduados del medio local que se sumen al programa.

- Participación institucional en eventos de emprendedorismo e innovación y en congresos de sistemas

- Talleres, como por ejemplo para elaboración de plan de negocios.

- Organización en la Facultad de la Semana Nacional del Emprendedor Tecnológico y ciclos de conferencias sobre marketing y otras temáticas.

- Presentación de emprendimientos mentoreados en el ecosistema emprendedor local. 
- Publicación de eventos propios e información de interés para los emprendedores en las redes sociales Facebook y Twitter.

- Encuentros de camaradería para difusión del programa e interrelación de los mentoreados.

- Difusión del programa de mentoreo en congresos nacionales (Guzmán et al., 2015), como así también en encuentros de docentes y estudiantes de U.T.N.

\section{Resultados obtenidos}

El año 2015 fue el inicio de este programa, donde se alcanzó a contener 10 proyectos de base tecnológica, conformado por 32 estudiantes y graduados, con el acompañamiento de 3 mentores y docentes de la Carrera Ingeniería en Sistemas de Información. Entre los principales logros de este período se destacan:

- Inicio de operaciones o "start-up" de dos emprendimientos en un espacio de trabajo compartido

- Obtención de un instrumento de financiamiento público, "Emprende INNdustria" (Gobierno de Córdoba, 2017), con la modalidad de "empresa madrina” y Premio provincial “Ideas Innovadoras 2015” de un emprendimiento

- Premio nacional “Sadosky 2015”, en la categoría inclusión digital, y Premio “Tec Microsoft 2015” de otro emprendimiento

Además dentro de este grupo, un estudiante descubrió su vocación docente y otro por la investigación científica. También se presentaron artículos sobre los emprendimientos y el programa en: el III Congreso Nacional de Ingeniería Informática y Sistemas de Información CONAIISI 2015, las Jornadas Argentinas de Tecnología, Innovación y Creatividad JATIC 2015 y el IX Congreso Nacional de Estudiantes de Ingeniería en Sistemas de Información CNEISI 2015.

La inserción de los proyectos en el ecosistema emprendedor local se realizó con la participación en dos eventos provinciales, la organización de la "III Semana Nacional del Emprendedor Tecnológico" en esta Facultad, el aporte de dos proyectos a la campaña de contenido social de la empresa Microsoft y la firma de dos convenios de colaboración, uno de ellos con una empresa de "coworking”, espacio de trabajo compartido, cuyos fundadores son graduados de la Carrera y el otro con una fundación de promoción a emprendimientos.

También se obtuvo la suscripción al programa "Microsoft BizSpark" para "startups" y se reforzaron lazos y se coordinaron acciones conjuntas con el grupo "UTN Emprende", de la carrera Ingeniería Industrial, y la incubadora de emprendimientos FIDE, dependiente de la Municipalidad de Córdoba, la Universidad Nacional de Córdoba y la Universidad Tecnológica Nacional.

En el segundo de ejecución de este programa se trabajó en el mentoreo de 23 proyectos, incluyendo a un total de 63 personas, entre estudiantes y graduados. Continuaron entre ellos emprendimientos del año anterior, incluidos los dos "starups", al que se le suma otro más que fue incubado por una aceleradora con sede en Córdoba y que le permitió una experiencia en Chile durante el 2015. Además 
dos de los proyectos son mentoreados en modalidad conjunta con el grupo "UTN Emprende" e integrantes de las Ingenierías en Sistemas de Información e Industrial.

Asumiendo nuevos desafíos, se efectuó la invitación a docentes y graduados de la Carrera para colaborar como mentores de los proyectos, mediante la cual se sumaron seis mentores al programa.

Los resultados más importantes del 2016 fueron: el "star-up" de siete nuevos emprendimientos, la continuidad los emprendimientos iniciados el año anterior, la obtención de un financiamiento del programa nacional FONSOFT, el primer premio para un emprendimiento de inclusión digital en la categoría 1 (Ideas o proyectos de soluciones) del Concurso Regional UIT-Samsung para América Latina y El Caribe "Aplicaciones móviles para la accesibilidad" recibido en México DF (ISIe, 2017), la publicación tres emprendimientos en el catálogo Innovar 2016 y la exposición de uno de ellos en Tecnópolis, la asignación de una beca para un programa de "start-ups" tecnológicos de Sillicon Valley y el acceso a otro programa para "startups" de una herramienta de "big data" de EE.U.U.

Los emprendimientos se presentaron en: el XVIII Workshop de Investigadores en Ciencias de la Computación WICC 2016, el Concurso Nacional de Innovaciones Innovar 2016, las Jornadas de Actualización en COPAIPA en Salta, la Competición Regional de la UIT para América Latina y el Caribe de Aplicaciones Móviles de Accesibilidad en México, Campus Party Argentina 2016 en Tecnópolis (ISIf, 2017). Además se obtuvo el aval institucional de una asociación civil de no videntes para un emprendimiento (U.Cor.Ci., 2017) y se llevó a cabo, en la Facultad, el lanzamiento de la segunda fase del emprendimiento MiAutobus.com y un ciclo de conferencias de marketing para emprendimientos tecnológicos innovadores. Estos eventos son de carácter gratuito y público. También el programa participó como colaborador del premio "MIT TechnologyReview en español", para innovadores menores de treinta y cinco años de Argentina y Uruguay, al cual se nominaron cinco emprendimientos (MIT, 2017), como organizador en la Regional de la "IV Semana Nacional del Emprendedor Tecnológico" y como expositor en la jornada "Edutech", que forma parte de la "Semana TIC de la Provincia de Córdoba". De esta manera se alcanzaron con creces los objetivos propuestos para el 2016.

\section{Conclusiones}

Mediante este programa, el Departamento de Ingeniería en Sistemas de Información ofrece, a estudiantes y graduados recientes de la Carrera, espacios y trayectos formativos que les permiten complementar su perfil profesional y tener una primera experiencia como emprendedores, partiendo de un trabajo grupal realizado en alguna de las cátedras.

Conscientes que en una asignatura de la currícula se dificulta desarrollar, con tiempo suficiente, el "espíritu emprendedor" o la "visión innovadora" de los estudiantes, se creó este espacio de trabajo colaborativo, opcional, con la modalidad de trabajo basada en el mentoreo. La función del mentor, a diferencia del profesor o del asesor, es de aprendizaje recíproco, ya que no sólo comparte sus conocimientos y experiencias con los mentoreados, sino que también aprende de ellos a partir de 
su involucramiento en los proyectos y en la elaboración de contenidos del negocio que se está iniciando. Esto basado en que dicho acompañamiento permite mejorar las posibilidades de éxito de los nuevos emprendimientos de base tecnológica que nacen de esta experiencia.

En vistas a las metas concretadas hasta la fecha y de la proyección para el año 2017, que cierra la primera edición del plan trianual del programa, se estima que se continuará con ediciones posteriores realizando las mejoras que indiquen las devoluciones de estudiantes, graduados y docentes de la Carrera. Además en conjunto con el programa "UTN Emprende" de Ingeniería Industrial de la misma Facultad, se propondrá extender las actividades de emprendedorismo al resto de las Ingenierías que estén convencidas de los beneficios académicos y profesionales, facilitando la conformación multidisciplinaria de los nuevos emprendimientos. 


\section{Referencias}

Angelelli, P. J., Prats, J. (2005). "Fomento de la actividad emprendedora en América Latina y el Caribe. Sugerencias para la formulación de proyectos" en Banco Interamericano de Desarrollo, Serie de informes técnicos del Departamento de Desarrollo Sostenible. Washington D.C.: 13, marzo.

Bentolila, C. et al. (2013). Emprende más. Herramientas para emprendedores en acción. Córdoba, Argentina: Ministerio de Industria, Comercio y Minería Gobierno de Córdoba.

Fracica Naranjo, G. (2009). "Formación del emprendedor universitario para el start up”, Grupo de Inva. Bogotá, Colombia: 11-12.

Gobierno de Córdoba - Ministerio de Industria, Comercio y Minería, Emprende Industria Córdoba, Extraído el 18/04/2017, Disponible en <http://www.cba.gov.ar/ emprende-inndustria-cordoba>.

González González, J.A., López Preciado, C.D. (2012). "El emprendimiento en los sistemas universitarios. El Tecnológico de Monterrey" en Serie Políticas Públicas y Transformación Productiva. Monterrey: CAF.

Guzmán, A.A., Pérez, N., Martin, S.M., Muñoz, R., Molina,C., Falcón,G. y Medina, O.C. (2015). "Programa de Mentoreo ISI" en Libro de Actas Jornadas Argentinas 2015 de Tecnología, Innovación y Creatividad (JATIC 2015), Organizador UCAECE, Mar del Plata, Buenos Aires, 4 al 6 de noviembre.

ISI a, Departamento de Ingeniería en Sistemas de Información de U.T.N. - F.R.C., Institucional - Novedades, Extraído el 18/04/2017. Disponible en <http://www. institucional.frc.utn.edu.ar/sistemas>.

ISI b, Departamento de Ingeniería en Sistemas de Información de U.T.N. - F.R.C., Mentoreo ISI, Extraído el 18/04/2017. Disponible en <https://www.facebook.com/ mentoreoisi>.

ISI c, Departamento de Ingeniería en Sistemas de Información de U.T.N. - F.R.C., Mentoreo ISI c, Extraído el 18/04/2017. Disponible en <https://twitter.com/ mentoreoisi>.

ISI d, Departamento de Ingeniería en Sistemas de Información de U.T.N. - F.R.C., Programa "Mentoreo de Emprendedores ISI", Extraído el 18/04/2017. Disponible en $<$ http://www.institucional.frc.utn.edu.ar/sistemas/Areas/noticias/Detalle. asp?1745>.

ISI e, Departamento de Ingeniería en Sistemas de Información de U.T.N. - F.R.C., CAECUS - Premiado con Beca para Participar de Evento en México, Extraído el 18/04/2017. Disponible en <http://www.institucional.frc.utn.edu.ar/sistemas/ Areas/noticias/Detalle.asp?1895>.

ISI f, Departamento de Ingeniería en Sistemas de Información de U.T.N. - F.R.C., 60 estudiantes y 3 emprendimientos de UTN-FRC participaron de Campus Party Argentina 2016 en Tecnópolis, Extraído el 18/04/2017. Disponible en <http://www. institucional.frc.utn.edu.ar/sistemas/Areas/noticias/Detalle.asp?1898>. 
Lederman, D., Messina, J., Pienknagura, S. y Rigolini, J. (2014). “E1 emprendimiento en América Latina: muchas empresas y poca innovación Resumen”. Washington, DC: Banco Mundial. Licencia: Creative Commons Attribution CC BY 3.0, págs. 5-6, Washington DC.

Microsoft a,"Microsoft Latinoamérica \#HaceGrandesCosas, Relevando Peligros en la vía pública", Extraído el 18/04/2017. Disponible en <https://www.youtube.com/ watch?v=W0rTsPgJTKQ>.

Microsoft b, "Microsoft Latinoamérica \#HaceGrandesCosas, STL Señas Uniendo 2 lenguajes", Extraído el 18/04/2017. Disponible en <https://www.youtube.com/ watch? $=$ =jvCduHF9QU>.

MIT, MIT TechnologyReview en español, Innovators Under 35 Argentina \& Uruguay 2016, Extraído el 18/04/2017. Disponible en <http://www. innovatorsunder35.com/innovators-under-35-argentina-uruguay $>$.

Paz, M., Grau, J. y Posso, F. (2014). "Análisis de las experiencias sobre formación de emprendedores en hispanoamérica", Acción Pedagógica, $\mathrm{N}^{\circ}$ 23: 60-73, enero diciembre.

U.Cor.Ci., Unión Cordobesa para Ciegos, "Caecus: Proyectos de tesis para ciegos", Extraído el 18/04/2017. Disponible en <http://ucorci.org.ar/caecus-proyectos-detesis-para-ciegos $>$. 